\title{
Lack of association between the CARD10 rs6000782 polymorphism and type 1 autoimmune hepatitis in a Japanese population
}

\author{
Kiyoshi Migita ${ }^{1,5^{*}}$, Yuka Jiuchi ${ }^{1}$, Hiroshi Furukawa ${ }^{2}$, Minoru Nakamura ${ }^{3}$, Atsumasa Komori ${ }^{1}$, Michio Yasunami ${ }^{4}$, \\ Hideko Kozuru', Seigo Abiru', Kazumi Yamasaki', Shinya Nagaoka', Satoru Hashimoto', Shigemune Bekki', \\ Kaname Yoshizawa' ', Masaaki Shimada ${ }^{1}$, Hiroshi Kouno ${ }^{1}$, Hiroshi Kamitsukasa', Tatsuji Komatsu', Taizo Hijioka', \\ Makoto Nakamuta', Atsushi Naganuma', Haruhiro Yamashita' ', Hideo Nishimura', Hajime Ohta', \\ Yoko Nakamura', Keisuke Ario', Yukio Oohara', Kazuhiro Sugi', Minoru Tomizawa', Takeaki Sato', \\ Hironao Takahashi ${ }^{1}$, Toyokichi Muro ${ }^{1}$, Fujio Makita' ${ }^{1}$ Eiji Mita ${ }^{1}$, Hironori Sakai ${ }^{1}$ and Hiroshi Yatsuhashi ${ }^{1}$
}

\begin{abstract}
Background: Previous genome-wide association studies have evaluated the impact of common genetic variants and identified several non-HLA risk loci associated with autoimmune liver diseases. More recent genome-wide association studies and replication analyses reported an association between variants of the CARD10 polymorphism rs6000782 and risk of type 1 autoimmune hepatitis (AlH). In this case-control study, we genotyped 326 Japanese AlH patients and 214 control subjects.

Results: Genomic DNA from 540 individuals of Japanese origin, including 326 patients with type-1 AlH and 214 healthy controls, was analyzed for two single nucleotide polymorphisms (SNPS) in the CARD10 gene. We selected CARD10 rs6000782 SNPs and genotyped these using PCR-RFLP method and direct sequencing. The Chi square test revealed that the rs6000782 variant alle (c) was not associated with the susceptibility for AlH in a Japanese population $[p=0.376$, odds ratio (OR) $1.271,95 \%$ confidence interval (Cl) $0.747-2.161]$ in an allele model. Our data also showed that CARD10 rs6000782 variants were not associated with AlH or with the clinical parameters of AlH.
\end{abstract}

Conclusions: In this study we examined an association between rs6000782 SNPs in the CARD10 gene and type-1 $\mathrm{AlH}$. Results showed no significant association of rs62000782 with type-1 AlH in a Japanese population. This study demonstrated no association between CARD10 rs6000782 variants and AlH in a Japanese population.

Keywords: Autoimmune hepatitis, Genetic factor, Genome-wide association study, CARD10

\section{Background}

Autoimmune hepatitis (AIH) is characterized by the presence of serum antibodies, both anti-nuclear (ANA) and anti-smooth muscle antibodies (ASMA), as well as elevated immunoglobulin G levels, and interface hepatitis [1]. The genetic factors underlying the occurrence of AIH are unknown, with the exception of certain human

\footnotetext{
${ }^{*}$ Correspondence: migita@nagasaki-mc.com

${ }^{5}$ Clinical Research Center, NHO Nagasaki Medical Center, Kubara

2-1001-1, Omura 856-8652, Japan

Full list of author information is available at the end of the article
}

leukocyte antigen (HLA) alleles [2]. de Boer et al. previously conducted a genome-wide association study that identified the most prominent association with $\mathrm{AIH}$ at rs2187668, which maps to the intronic region of $H L A-$ $D Q A 1$ [3]. They also showed that AIH was associated with variants of genes encoding Scr homology 2 adaptor protein 3 (SH2B3; rs3184504) and caspase recruitment domain-containing protein 10 (CARD10; rs6000782) [3]. In view of the importance of understanding the contribution of genetics to $\mathrm{AIH}$, we carried out a case-control study to investigate the association between variants 
of CARD10 rs6000782 and type $1 \mathrm{AIH}$ in a Japanese population.

\section{Methods}

\section{Study population}

Consecutive type $1 \mathrm{AIH}$ patients $(\mathrm{n}=326)$ diagnosed according to the international diagnostic criteria for $\mathrm{AIH}$ [4] from the register of the Japanese National Hospital Organization (NHO) Liver Network Registry beginning in 2009 were enrolled in the present study as a multicenter cohort population [5]. Patients exhibiting primary biliary cirrhosis were excluded from the analysis. As controls, 214 healthy Japanese subjects (74 men and 140 women, with a mean age of $47.5 \pm 10.8$ years) with no history of liver disease were also enrolled. All patients did not have any other types of liver diseases such as chronic hepatitis $C$, alcoholic liver diseases, autoimmune liver diseases, or metabolic liver diseases. This study was conducted by adhering to the STOROBE statement (casecontrol studies). The study protocol was approved by the Ethics Committees of National Nagasaki Medical Center, and written informed consent was obtained from each individual.

\section{DNA extraction and genotyping}

Blood samples were taken from all study participants, and genomic DNA was isolated from peripheral blood leukocytes using a DNA blood mini kit from Qiagen (Hilden, Germany) according to the manufacturer's guidelines. $C A R D 10$ rs6000782 genotypes were determined by the polymerase chain reaction-restriction fragment length polymorphism (PCR-RFLP) method. For the sequence (Fig. 1a), PCR products were treated with ExoSAP-IT (Affymetrix, Inc., Santa Clara, CA), and then sequenced using a BigDye Terminator v1.1 Cycle Sequencing Kit (Life Technologies, Tokyo, Japan). Sequences were analyzed using an Applied Biosystems 3130xl Genetic Analyzer (Life Technologies). Restriction fragment length polymorphism analysis was performed after the identification of single nucleotide polymorphism-specific restriction sites by NCBI Entrez SNP (http://www.ncbi. nlm.nih.gov/snp) and Takara Cut-Site Navigator (http:// www.takara-bio.co.jp/enzyme). PCR restriction fragment length polymorphism genotyping to detect the 37928186A > C base pair change was performed using the following cycling profile: $95^{\circ} \mathrm{C}$ for $5 \mathrm{~min}$, followed by 35 cycles at $95^{\circ} \mathrm{C}$ for $1 \mathrm{~min}, 56^{\circ} \mathrm{C}$ for $1 \mathrm{~min}$, and $72^{\circ} \mathrm{C}$ for $1 \mathrm{~min}$. The 365 -base pair product was digested with $X s p \mathrm{I}$ at $37^{\circ} \mathrm{C}$ for $6 \mathrm{~h}$ and analyzed by $4 \%$ NuSieve 3:1 agarose gel electrophoresis (Fig. 1b).

\section{Statistical analyses}

Results are expressed as mean \pm SD. The statistical significance of differences between groups was calculated by either the Chi square test or Fisher's exact test for categorical data and Mann-Whitney's U test for quantitative data. Multivariate logistic regression analysis was performed with SPSS v.18 for windows (SPSS Statistics, Illinois). Deviation from Hardy-Weinberg equilibrium was assessed using the SNPAlyze software ver. 7.0 (Dynacom,

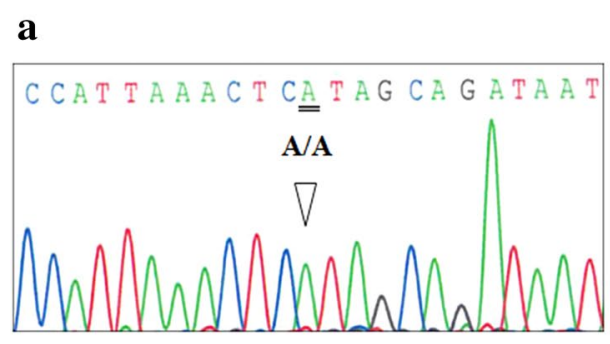

b
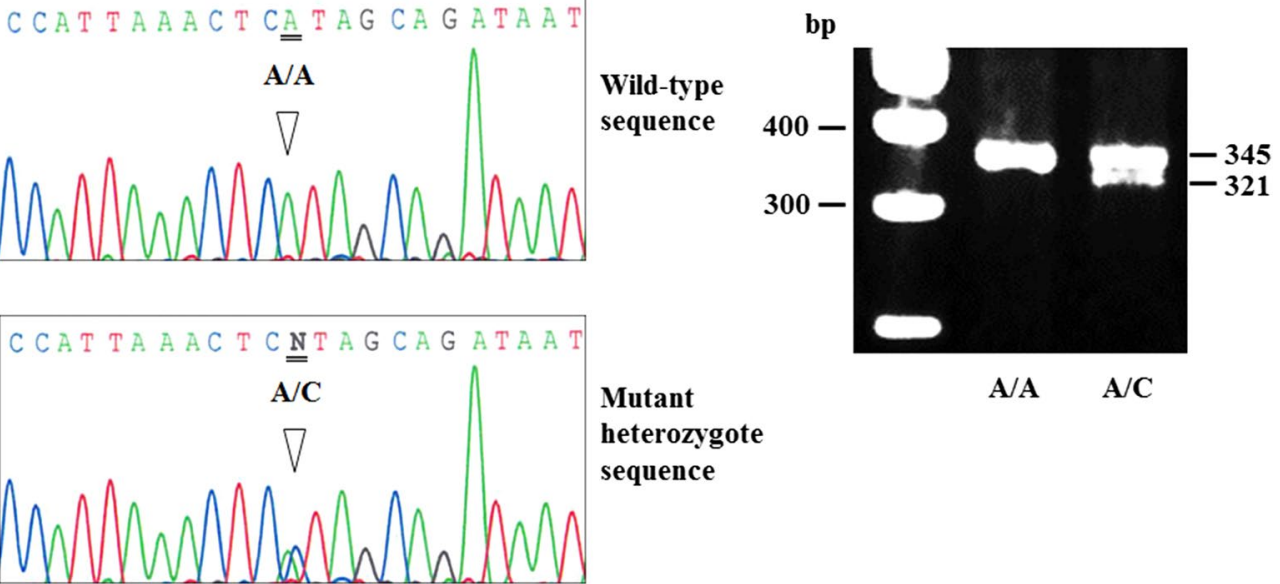

Fig. 1 Genotyping of CARD10 rs6000782. a CARD10 rs6000782 is positioned 12,643 base pairs downstream in the $22 \mathrm{q} 13.1$ region. For sequencing primers, forward primer 5'-TTGAGACGGGGTCTCGCT-3' and reverse primer 5'-GCCAAACCCGAGGTAATCTA-3'. b The SNP CARD10 rs6000782 A/C were genotyped from a PCR fragment with an average size of $365 \mathrm{bp}$. The dimorphism rs6000782 A/C was typed by RFLP method, the amplified PCR product was digested with Xspl restriction enzyme, resulting in 321 and 24 and 20 bp fragments for allele C and an intact fragment of 345 and $20 \mathrm{bp}$ for allele $\mathrm{A}$. The small fragments (<50 bp) are not visible on the gel 
Yokohama, Japan). A $p$ value of $<0.05$ was considered significant.

\section{Results}

\section{Demographic data of patients with AlH}

Table 1 presents the demographic data of the subjected AIH patients. Among the enrolled type-1 AIH patients, 288 (88.6 \%) were positive for ANA (>1:40) and 121 (38.2 \%) for ASMA (>1:40). Among 326 eligible patients,

Table 1 Baseline characteristics of 326 Japanese AlH type 1 patients

\begin{tabular}{ll}
\hline Characteristics & $\mathbf{N}=\mathbf{3 2 6}(\%)$ \\
\hline Female, n/total (\%) & $289 / 326(88.7)$ \\
Age, years, mean \pm SD & $59.5 \pm 13.3$ \\
Biochemistry & \\
AST, IU/L, median (IQR) & $255.0(92.5-723.0)$ \\
ALT, IU/L, median (IQR) & $297.0(104.5-818.0)$ \\
ALP, IU/L, median (IQR) & $432.0(316.5-584.0)$ \\
Total Bilirubin, mg/dl, median (IQR) & $1.3(0.8-4.6)$ \\
Albumin, g/dl, median (IQR) & $3.9(3.5-4.2)$ \\
IgG, mg/dl, median (IQR) & $2239.0(1809.5-2390.0)$ \\
Platelets, 104/Ml, median (IQR) & $18.5(13.9-23.0)$ \\
Serology & \\
ANA $\geqq 1: 40, n /$ total (\%) & $288 / 325(88.6)$ \\
ASMA $\geqq 1: 40, n /$ total (\%) & $121 / 317(38.2)$ \\
Histology & \\
Cirrhosis, n/total (\%) & $51 / 326(15.6)$ \\
IAlHG criteria & \\
Score, median (IQR) & $16(14-18)$ \\
Probable AlH, n/total (\%) & $126 / 309(40.8)$ \\
Definite AlH, n/total (\%) & $183 / 309(59.2)$ \\
\hline
\end{tabular}

AST aspartate aminotransferase, $A L T$ alanine aminotransferase, $A L P$ alkaline phosphate, $\lg G$ immunoglobulin G, ANA anti-nuclear antibody, ASMA anti-smooth muscle antibody, IQR interquartile range, IAIHG International Autoimmune Hepatitis Group
35 (10.7 \%) had liver cirrhosis at the time of diagnosis, and among the remaining 291 patients without liver cirrhosis, 16 developed liver cirrhosis during the follow-up.

\section{Association of CARD10 polymorphisms with type-1 AlH}

Genotype distributions were in Hardy-Weinberg equilibrium in cases and controls (Table 2). Genotype frequencies and distributions, as well as odds ratios (ORs) and $95 \%$ confidence intervals (CIs) for the association with AIH are shown in Table 3. The rs6000782 C allele was shown not to be associated with an increased risk for AIH (OR 1.271; 95 \% CI 0.747-2.161; $p=0.376$ ).

We also performed a detailed genotype-phenotype analysis using the clinical data. A detailed genotype-phenotype analysis using the clinical data revealed no significant association between rs6000782 and clinical findings of AIH patients (Table 4).

\section{Discussion}

AIH is characterized by an imbalanced regulation of the immune system in which innate and adaptive immune responses to hepatocyte antigens are important [6]. Genetic variation in the immune mechanisms that establish and maintain self-tolerance is likely to play a role in the development of AIH [7]. The susceptibility genes of AIH act alone or with environmental factors whose identity is mostly unknown [8]. The strongest association is with genes located within the HLA region, particularity those encoding the HLA-DRB1 alleles [2]. Up until now, evaluation of the non-HLA genetics of AIH has focused on small scale (usually non-replicated) candidate gene studies [9]. Genome-wide screening is a promising approach for the identification of the genetic determinants of complex diseases [8]. Large case-control studies with genome-wide surveys of genetic risk have been demonstrated for primary biliary cirrhosis (PBC) $[10,11]$. $\mathrm{AIH}$ was subjected to the a similar genome-wide survey.

Table 2 Frequencies of CARD10 rs6000782 genotypes

\begin{tabular}{|c|c|c|c|c|c|}
\hline & Locus & Genotype & Observed number (\%) & $\begin{array}{l}\text { Expected } \\
\text { number }^{\mathrm{a}}\end{array}$ & $p$ value $^{b}$ \\
\hline \multicolumn{6}{|c|}{ Patients with $\mathrm{AlH}$} \\
\hline \multirow[t]{3}{*}{$n=326$} & rs6000782 & $\mathrm{A} / \mathrm{A}$ & $284(87.1)$ & 285.3 & $x^{2}=1.746$ \\
\hline & & $\mathrm{A} / \mathrm{C}$ & $42(12.9)$ & 39.3 & $p=0.523$ \\
\hline & & $\mathrm{C} / \mathrm{C}$ & 0 & 1.4 & \\
\hline \multicolumn{6}{|c|}{ Healthy control } \\
\hline \multirow[t]{3}{*}{$n=214$} & rs6000782 & $\mathrm{A} / \mathrm{A}$ & $193(90.2)$ & 192.5 & $x^{2}=0.280$ \\
\hline & & $\mathrm{A} / \mathrm{C}$ & $20(9.3)$ & 20.9 & $p=1.000$ \\
\hline & & $\mathrm{C} / \mathrm{C}$ & $1(0.5)$ & 0.6 & \\
\hline
\end{tabular}

\footnotetext{
${ }^{a}$ Expected genotype frequencies based on observed allele frequencies and assuming Hardy-Weinberg equilibrium

b $p$ values were calculated using the Chi square test for Hardy-Weinberg equilibrium at individual loci
} 
Table 3 CARD10 rs6000782 polymorphism in patients with type 1 AlH and healthy controls

\begin{tabular}{|c|c|c|c|c|}
\hline & $\begin{array}{l}\text { Healthy } \\
\text { control (\%) } \\
n=214\end{array}$ & $\begin{array}{l}\text { AlH (\%) } \\
n=326\end{array}$ & $p$ value $^{a}$ & OR $(95 \% \mathrm{Cl})$ \\
\hline $\begin{array}{l}\text { Genotype } \\
\text { frequencies }\end{array}$ & & & 0.186 & \\
\hline $\mathrm{A} / \mathrm{A}$ & $193(90.2)$ & $284(87.1)$ & & \\
\hline $\mathrm{A} / \mathrm{C}$ & $20(9.3)$ & $42(12.9)$ & & \\
\hline $\mathrm{C} / \mathrm{C}$ & $1(0.5)$ & 0 & & \\
\hline $\begin{array}{l}\text { Allele frequen- } \\
\text { cies }\end{array}$ & & & 0.376 & \\
\hline A & 406 (94.9) & 610 (93.6) & & 1 \\
\hline C & $22(5.1)$ & $42(6.4)$ & & $\begin{array}{l}1.271(0.747- \\
2.161)\end{array}$ \\
\hline
\end{tabular}

OR odds ratio, $\mathrm{Cl}$ confidence interval

a Genotype frequencies were determined by $x^{2}$ test using $2 \times 3$ contingency tables between patients with $\mathrm{AlH}$ and healthy controls. Allele frequencies were determined by $\mathrm{x}^{2}$ test using $2 \times 2$ contingency tables between patients with $\mathrm{AlH}$ and healthy controls

The first application for type 1 AIH by de Boer et al. in AIH patients identified three genes exhibiting significant association in 649 patients and 13,436 healthy controls in Dutch and German populations. The main finding of this study was the strength of the AIH association with HLA, although it also identified associations with the $\mathrm{SH} 2 \mathrm{B3}$ rs3184504*A allele and CARD10 rs6000782 *C allele.

The present study found no association of CARD10 rs6000782 variants with type $1 \mathrm{AIH}$ in a Japanese population. The major strength of this study is the finding that the association discovers by deBoer in a Caucasian Northern European Dutch and German population is not generalizable to the East Asian Japanese population. While this contrasts with the finding of de Boer et al. in a Caucasian population, it is in agreement with the documented lack of association between CARD10 variants and $\mathrm{AIH}$ in populations of diverse racial backgrounds. Gene-gene interactions or epistasis have been proposed to occur between genes that cluster within specific immune pathways, thus enhancing their effect on disease susceptibility [12]. These have been reported in autoimmune diseases, including a possible interaction between HLA and non-HLA genes [13]. This suggests that further gene-gene interaction studies will be necessary to determine the associations of different susceptibility loci in AIH.

The main finding of the study by de Bore et al. [3] remains the relative strength of the HLA associations. The associations of AIH with variants of $\mathrm{SH} 2 \mathrm{B3}$ rs3184504 $\left(p=7.7 \times 10^{-8}\right)$ and CARD10 rs6000782 $\left(p=3.0 \times 10^{-6}\right)$ did not reach the accepted level of significance required to declare genome-wide significance $\left(p<5 \times 10^{-8}\right)$. However, the prior association of SH2B3 variants with autoimmune diseases suggests that this association is likely to be confirmed with larger cohorts. We did not analyze the association of SH2B3 rs3184504 and $\mathrm{AIH}$ in our current study because it is almost monomorphic in a Japanese population (http://www.ncbi.nlm. nih.gov/projects/SNP/snp_ref.cgi?rs=3184504). However, such validation is necessary because attempts to

Table 4 Comparison of demographics between AlH patients with or without rs6000782 C allele

\begin{tabular}{|c|c|c|c|}
\hline & $\begin{array}{l}\text { rs6000782 C allele }(+) \\
n=42\end{array}$ & $\begin{array}{l}\text { rs6000782 C allele (-) } \\
n=284\end{array}$ & $p$ \\
\hline Female, n (\%) & $38(90.5)$ & $251(88.4)$ & 0.465 \\
\hline Age, years, mean $\pm S D$ & $59.6 \pm 13.9$ & $59.5 \pm 13.3$ & 0.786 \\
\hline \multicolumn{4}{|l|}{ Biochemistry } \\
\hline AST, IU/L [median (IQR)] & $189.0(85.5-835.0)$ & $264.0(94.0-707.5)$ & 0.620 \\
\hline ALT, IU/L [median (IQR)] & $258.5(93.0-906.0)$ & $305.0(104.5-813.0)$ & 0.926 \\
\hline ALP, IU/L [median (IQR)] & $402.5(261.0-566.0)$ & $437.0(323.5-586.5)$ & 0.242 \\
\hline Total bilirubin, mg/dl [median (IQR)] & $1.9(0.8-4.6)$ & $1.2(0.8-4.6)$ & 0.680 \\
\hline Albumin, g/dl [median (IQR)] & $3.9(3.4-4.2)$ & $3.9(3.5-4.2)$ & 0.844 \\
\hline $\operatorname{lgG}, \mathrm{mg} / \mathrm{dl}[$ median (IQR)] & $2078.0(1695.0-2691.5)$ & $2250.0(1835.5-2945.0)$ & 0.199 \\
\hline Platelets, $10^{4} / \mu \mathrm{l}$ [median (IQR)] & $19.6(15.1-22.4)$ & $18.4(13.9-23.1)$ & 0.712 \\
\hline \multicolumn{4}{|l|}{ Serology } \\
\hline ANA $\geqq 1: 40, n(\%)$ & $36(85.7)$ & $252(89.0)$ & 0.339 \\
\hline$A S M A \geqq 1: 40, n(\%)$ & $13(31.0)$ & $108(39.3)$ & 0.301 \\
\hline \multicolumn{4}{|l|}{ Histology } \\
\hline Cirrhosis, n (\%) & $8(19.0)$ & $43(15.1)$ & 0.515 \\
\hline
\end{tabular}

AST aspartate aminotransferase, $A L T$ alanine aminotransferase, $A L P$ alkaline phosphate, $l g G$ immunoglobulin G, $A N A$ anti-nuclear antibody, $A S M A$ anti-smooth muscle antibody, IQR interquartile range 
generalize genetic associations across ethnicities have had mixed results. For CARD10 rs6000782, more validation studies are warranted. Our study only enrolled patients with a definite diagnosis of $\mathrm{AIH}$, in order to prevent any potential case ascertainment bias. This approach limited the sample size and therefore introduced the limitation of reduced statistical power, which might have prevented us from identifying potential associations between CARD10 rs6000782 and AIH.

\section{Conclusions}

Our findings showed that CARD10 rs6000782 is not likely to be associated with type $1 \mathrm{AIH}$, at least in a Japanese population. However, because allele frequency distributions differ according to ethnicity, replication in other populations and functional studies should be initiated in order to clarify the contribution of this genetic background in the development of AIH . Genetic variations associated with AIH susceptibility remain for further investigation.

\section{Abbreviations}

AlH: autoimmune hepatitis; CARD10: caspase recruitment domain-containing protein 10; HLA: human leukocyte antigen; NHO: National Hospital Organization; SH2B3: SH2B adaptor protein 3; SNPs: single nucleotide polymorphisms.

\section{Authors' contributions}

KM carried out the molecular genetic studies, participated in the sequence alignment and drafted the manuscript. YJ, HKoz, MY participated in the sequence alignment. AK, SA, KYa, SN, SH, SB, KYo, MS, HKou, HKa, TK, TH, MN, AN, HY, HN, HO, YN, KA, YO, KS, MT, TS, HT, TM, FM, EM, HS, HY collected the clinical data. HF, MN, participated in the design of the study and performed the statistical analysis. All authors read and approved the final manuscript.

\footnotetext{
Author details

${ }^{1} \mathrm{NHO}-\mathrm{AlH}$ study group, Nagasaki Medical Center, Kubara 2-1001-1, Omura, Nagasaki 856-8562, Japan. ${ }^{2}$ Department of Rheumatology, NHO Sagamihara Hospital, Minamikusakuradai 18-1, Sagamihara, Kanagawa 252-0392, Japan. ${ }^{3}$ Department of Hepatology, Nagasaki University Graduate School of Biomedical Sciences, Sakamoto 1-12-4, Ngasaki, Nagasaki 852-8523, Japan. ${ }^{4}$ Department of Clinical Medicine, Institute of Tropical Medicine, Nagasaki University, Sakamoto 1-12-4, Ngasaki, Nagasaki 852-8523, Japan. ${ }^{5}$ Clinical Research Center, NHO Nagasaki Medical Center, Kubara 2-1001-1, Omura 856-8652, Japan.
}

\section{Acknowledgements}

This study was supported by a grant from the National Hospital Organization (Liver-net work study).

\section{Competing interests}

The authors declare that they have no competing interests.

Received: 15 April 2015 Accepted: 23 November 2015

Published online: 12 December 2015

\section{References}

1. Czaja AJ, Manns MP. Advances in the diagnosis, pathogenesis, and management of autoimmune hepatitis. Gastroenterology. 2010;139:58-72.

2. Oliveira LC, Porta G, Marin ML, Bittencourt PL, Kalil J, Goldberg AC. Autoimmune hepatitis HLA and extended haplotypes. Autoimmun Rev. 2011;10:189-93.

3. de Boer YS, van Gerven NM, Zwiers A, et al. Genome-wide association study identifies variants associated with autoimmune hepatitis type 1. Gastroenterology. 2014;147:443-52.

4. Alvarez F, Berg PA, Bianchi FB, et al. International Autoimmune Hepatitis Group Report: review of criteria for diagnosis of autoimmune hepatitis. J Hepatol. 1999;31:929-38.

5. Migita K, Watanabe Y, Jiuchi Y, et al. Hepatocellular carcinoma and survival in patients with autoimmune hepatitis (Japanese National Hospital Organization-autoimmune hepatitis prospective study). Liver Int. 2012;32:837-44

6. Heneghan MA, Yeoman AD, Verma S, Smith AD, Longhi MS. Autoimmune hepatitis. Lancet. 2013;382:1433-44.

7. Czaja AJ, Donaldson PT. Genetic susceptibilities for immune expression and liver cell injury in autoimmune hepatitis. Immunol Rev. 2000;174:250-9

8. Mells GF, Kaser A, Karlsen TH. Novel insights into autoimmune liver diseases provided by genome-wide association studies. J Autoimmun. 2013;46:41-54.

9. Tang J, Zhou C, Zhang ZJ, Zheng SS. Association of polymorphisms in non-classic MHC genes with susceptibility to autoimmune hepatitis. Hepatobiliary Pancreat Dis Int. 2012;11:125-31.

10. Mells GF, Floyd JA, Morley KI, Cordell HJ, Franklin CS, Shin SY, et al. Genome-wide association study identifies 12 new susceptibility loci for primary biliary cirrhosis. Nat Genet. 2011;43:329-32.

11. Nakamura M, Nishida N, Kawashima M, Aiba Y, Tanaka A, Yasunami M, et al. Genome-wide association study identifies TNFSF15 and POU2AF1 as susceptibility loci for primary biliary cirrhosis in the Japanese population. Am J Hum Genet. 2012;91:721-8.

12. Wei $W H$, Hemani G, Haley CS. Detecting epistasis in human complex traits. Nat Rev Genet. 2014;15:722-33.

13. Parkes M, Cortes A, van Heel DA, Brown MA. Genetic insights into common pathways and complex relationships among immune-mediated diseases. Nat Rev Genet. 2013;14:661-73.

Submit your next manuscript to BioMed Central and we will help you at every step:

- We accept pre-submission inquiries

- Our selector tool helps you to find the most relevant journal

- We provide round the clock customer support

- Convenient online submission

- Thorough peer review

- Inclusion in PubMed and all major indexing services

- Maximum visibility for your research

Submit your manuscript at www.biomedcentral.com/submit

() Biomed Central 